\title{
L'invention et la réinvention de la Nouvelle Muséologie
}

Invention and reinvention of "New Museology" (nouvelle museologie)

La invención y la reinvención de la Nueva Museología

\section{Bruno Brulon Soares}

\section{OpenEdition}

Journals

Édition électronique

URL : https://journals.openedition.org/iss/563

DOI : $10.4000 /$ iss. 563

ISSN : 2306-4161

\section{Éditeur}

ICOM - International Council of Museums

Édition imprimée

Date de publication : 1 juin 2015

Pagination : $57-72$

ISSN : 2309-1290

\section{Référence électronique}

Bruno Brulon Soares, "L'invention et la réinvention de la Nouvelle Muséologie », ICOFOM Study Series [En ligne], 43a | 2015, mis en ligne le 06 février 2018, consulté le 16 février 2023. URL : http:// journals.openedition.org/iss/563; DOI : https://doi.org/10.4000/iss.563 


\title{
L'invention et la réinvention de la Nouvelle Muséologie
}

\author{
Bruno Brulon Soares
}

\author{
Université Fédérale de l'État de Rio de Janeiro - UNIRIO, \\ Brésil
}

En 1984, au moment de la configuration du Mouvement International pour une Nouvelle Muséologie (MINOM), André Desvallées ${ }^{26}$ déclarait que "dans le Comité de muséologie, il ne pouvait être question que d'une seule muséologie, ni vieille, ni nouvelle " (Desvallées, 1985, p. 69). C'était le moment de la légitimation d'un mouvement pour la rénovation de la muséologie, déjà en cours pendant les années 1970. Les dirigeants du MINOM demandaient qu'il soit reconnu comme organisation associée du Conseil International des Musées (ICOM). Desvallées a été invité par le bureau de l'ICOM pour témoigner, en tant que membre du Comité international de Muséologie, l'ICOFOM, sur l'importance de ce mouvement. Avec une position stratégique dans ce premier moment de la constitution d'un champ scientifique et politique de la muséologie à l'ICOM, Desvallées a légitimé le mouvement d'une rupture présumée et, en même temps, il a rendu possible l'absorption de ce même mouvement au sein de l'ordre muséologique établi.

À la suite de cette communication, le Conseil exécutif de l'ICOM prit une décision de compromis, en acceptant comme organisation associée le MINOM, qui s'était créé entre temps, mais en se refusant à créer un comité spécifique pour les écomusées au sein de l'organisation (Desvallées, 2014 27 ). L'ICOFOM a continué dans la poursuite d'une muséologie plus réflexive et critique, mais, progressivement, les dialogues avec le MINOM sont devenus plus difficiles et limités. Avec son internationalisation plus vaste, le MINOM a créé une nouvelle tendance pour la muséologie, en profitant des idées de la nouvelle muséologie issues du contexte français.

L'ICOFOM et le MINOM, les deux forums observés dans ce travail, montrent les différents modes d'appropriation des mêmes idées originelles. Si l'ICOFOM a travaillé, influencé par des idées de la nouvelle muséologie, vers l'évolution de la discipline comme un ensemble (de questions, de sujets, et, finalement, de concepts), le MINOM, au contraire, dans une certaine mesure, a diffusé l'idée d'une muséologie fragmentée, entre l'ancienne et la nouvelle, mais, plus récemment, entre une branche d'une " muséologie sociale » (ou " sociomuséologie », en certains contextes) et l'autre muséologie, moins concernée par les problèmes sociaux. Centré sur le travail pratique dans les musées, cette "muséologie sociale » se présente

\footnotetext{
${ }^{26}$ Conservateur général honoraire du Patrimoine en France. En tant que membre du Conseil international des musées (ICOM) depuis 1966, André Desvallées (1931- ) fut membre du Bureau de son Comité français de 1981 à 1995 et, membre du Comité international pour la muséologie (ICOFOM), il en fut Secrétaire de 1980 à 1983 puis Vice-président de 1983 à 1998 et, depuis 2001, conseiller permanent. Inventeur du concept de la "nouvelle muséologie" (1980), il a été en outre membre fondateur des associations "Muséologie nouvelle et expérimentation sociale" et "Musées, hommes, société" en France, et du MINOM (1984), sur le plan international.

${ }^{27}$ Communication par e-mail. 28 novembre, 2013.
} 
moins comme un courant théorique, et plus comme une idéologie du musée (instrument social). Ses acteurs réinventent la «nouvelle muséologie » dans le présent, à partir d'une série de propositions posées au début du mouvement : l'idée du « musée social » balisé par la notion du « musée intégral » (1972) qui s'est propagée dans presque toute l'Amérique Latine, le concept d'une "muséologie communautaire ", parente de l'écomuséologie qui s'est développée au musée du Creusot pendant les années 1970, l'opposition entre une "muséologie active »(1984) et la muséologie " officielle » (passive ?), etc.

Quant à la muséologie qui s'est exprimée dans le monde depuis les années 1980, on a, maintenant, encore quelques questions sur le changement réel qu'elle a proposé à l'univers muséal. Quels ont été ses vrais impacts sur la muséologie " officielle »? Qu'est-ce qui a effectivement changé? Et quelle est la muséologie du présent, comprise en tant que champ d'étude plus au moins défini ?

\section{L’invention de la nouveauté au sein des musées}

En tant qu'établissement qui, au long de la Modernité européenne, a été constamment associé au passé (Brulon Soares, 2011), le musée a changé l'image, construite historiquement par lui-même, d'un lieu dont le but serait la fixation des choses du réel, pour être pensé comme un endroit de rencontres entre des expériences. Grâce au développement d'une muséologie contemporaine engagée avec une nouvelle pratique associée à la proposition contestataire d'un " nouveau musée ", les années 1970 sont marquées, en France, par l'épanouissement d'une nouvelle mentalité chez un certain nombre de professionnels des musées. Toutefois, le point de départ d'une « muséologie nouvelle » est encore indéfini.

On peut envisager que le mouvement de décolonisation des mentalités en Europe, dans la seconde moitié du $X X^{e}$ siècle, à partir du contact plus courant avec des idées développées dans les colonies, a soulevé une "vague ${ }^{28}$ " de nouveautés qui ont atteint le champ muséal. Dans les années 1960, après l'indépendance de beaucoup de ces pays et lorsqu'ont éclaté divers mouvements sociaux dans le monde, un secteur de la muséologie internationale a commencé à percevoir les musées différemment. Peut-être l'évènement le plus important qui a témoigné de ce changement progressif des mentalités sur les musées a-t-il été la $9^{\text {ème }}$ Conférence Générale de l'ICOM, à Paris, Dijon et Grenoble.

Organisée par le Comité français de l'ICOM, entre le 29 août et le 10 septembre 1971, la Conférence qui a réuni plus de six cents participants, répartis entre les 56 pays représentés (Nouvelles de I'ICOM, 1971), avait comme thème de discussion: "Le musée au service de l'homme, aujourd'hui et demain - le rôle éducatif et culturel du musée ». Comme le remarque Desvallées (1992, p. 17), cet évènement a été le « point de départ international » des idées qui ont, plus tard, servi de principes de base à la nouvelle muséologie. Ce n'est pas, bien sûr, la première fois que l'ICOM s'est intéressé à un thème qui relie le musée à la société. En effet, à partir de la nomination de Georges-Henri Rivière en tant que premier directeur de I'ICOM, de 1948 à1965, l'intention de développer des pratiques

\footnotetext{
${ }^{28}$ Terme utilisé par André Desvallées pour intituler les deux volumes des textes fondateurs de la «nouvelle muséologie » (Desvallées, De Barry \& Wasserman, 1992 et 1994).
} 
muséales plus ouvertes et 'inclusives' était déjà claire ${ }^{29}$. C'est encore à Dijon, lors de cette même conférence, le 3 septembre, que le terme « écomusée » a été prononcé en public pour la première fois, par Robert Poujade, maire de la ville et alors ministre de l'Environnement.

Quelques "grands noms » de la muséologie internationale sont intervenus pendant la Conférence, parmi lesquels Duncan $F$. Cameron $^{30}$ (Canada), John Kinard (États-Unis), créateur d'un musée de voisinage à Washington D.C., Mario Vásquez (Mexique), créateur du musée d'Anthropologie de Mexico et Stanislas Adotevi (Dahomey) qui, dans une intervention corrosive, remit en cause tous les fondements du musée. Les déclarations d'Adotevi ont dû plus déranger que la phrase de Jean Chatelain, alors Directeur des Musées de France et Président du Comité français de I'ICOM, énonçant « $A u$ Louvre, nous n'avons pas besoin d'animation, nous avons la Joconde et la Vénus de Milo » (Desvallées, 1992, p. 17).

La conférence d'Adotevi, le 5 septembre 1971, en ouverture de la première séance plénière de la Conférence, présidée par Rivière, a été ensuite analysée par le groupe de travail pour la discussion des séances plénières, en raison de la "valeur de choc » (Rivière et al., 1971 , p. 1) des thèses présentées. Rivière a conclu que, selon cette conférence :

De très nombreux musées - et non des moindres - ne sont plus adaptés aujourd'hui aux conditions de vie et à la pensée de l'homme contemporain. Ils se présentent en effet comme les héritiers d'une longue tradition européenne, qui n'a servi jusqu'ici que les intérêts d'une minorité (Rivière et al., 1971, p. 1).

Envisagé comme un établissement qui « doit prendre, face aux finalités de son action, une attitude constamment critique " (Rivière et al., 1971, p. 2), le « nouveau musée » qui est proposé à ce moment est le résultat d'une "transformation radicale ". Le rêve de certains Européens touchés par les idées de penseurs africains comme Stanislas Adotevi ou américains comme Mario Vásquez et John Kinard avait déjà commencé à se développer comme une réalité pour les différents contextes où une muséologie renouvelée était en train d'apparaître en tant que pratique expérimentale. Entre tous ces porteurs de nouvelles idées, c'est Rivière, en tant que Conseiller permanent de l'ICOM, qui va lancer une première réflexion à partir de ce confluent d'idées, déconcertantes pour la muséologie à laquelle s'accrochaient les «anciens dominants " "d'un champ de savoirs euro-centrique ${ }^{32}$. Au même moment, Hugues de Varine, son

\footnotetext{
${ }^{29}$ Ces tendances ont marqué surtout les années 1950, quand les nouvelles théories de l'éducation et les musées scandinaves influencèrent la muséologie - notamment lors de la Conférence Générale de l'ICOM de 1956, à Genève. Les années 1960 sont marquées par l'adoption par l'ICOM d'un programme plus orienté vers l'environnement, lors de la Conférence Générale de 1962, à La Haye et Amsterdam, sur le développement des musées locaux et régionaux. (Baghli, Boylan, \& Herreman, 1998, p.45).

${ }^{30}$ Pour sa part, c'est dans cette même année que Duncan Cameron remit en cause le système de communication du musée, qui accroît son caractère élitiste. II souhaitait que le musée soit aussi le lieu de la contestation, un «forum », et pas seulement un " temple » (Cameron, 1971).

${ }^{31}$ Dans le sens donné par Pierre Bourdieu (1976, p. 96).

${ }^{32}$ La position de Rivière dans ce champ euro-centrique n'était pas, néanmoins, une position dominante par rapport à la muséologie hégémonique structurée dans les idées des professionnels des musées traditionnels européens. Depuis la création de I'ICOM, en 1946, en tant que son premier directeur, Rivière a assuré une plus grande dissémination des idées sur les musées dans les différentes régions du monde,
} 
successeur à la direction de l'ICOM, bousculera encore davantage les idées reçues de la profession.

A la suite de la Conférence de 1971, d'autres rencontres ont été décisives pour la diffusion des idées initiales d'un mouvement à venir. L'année 1972 est marquée par la célèbre table ronde, en maijuin, à Santiago-du-Chili, organisée par l'UNESCO et intitulée Rôle du Musée dans l'Amérique latine, qui a été le moment de diffusion internationale des questions initiales lancées en France en 1971, et le colloque organisé par l'ICOM et intitulé Musée et environnement, entre le 25 et le 30 septembre, en France, à Istre et à Lourmarin, dans lequel le terme écomusée a commencé à gagner un contenu.

D'autres moments et évènements antérieurs dénotent le large éventail des transformations au sein des expériences muséales. Aux États-Unis, on peut remonter à la fin des années 1960 et à l'idée du neighborhood museum ("musée de voisinage »), conçue d'abord par John Kinard, fondateur en 1967 du Neighborhood Museum dans le quartier d'Anacostia à Washington, où la communauté locale s'est approprié un musée classique pour penser les problèmes urbains partagés par le groupe (Kinard \& Nighbert, 1972). Au même moment, les Children's museums ("musées des enfants»), créés au Brooklyn Museum, accentuaient l'importance de l'éducation muséale dans les nouvelles générations. Au Mexique, dans les années 1960, Mario Vásquez expérimenta la Casa del Museo, en envisageant la mise en valeur des mœurs préhispaniques dans la vie des habitants locaux. Au Canada, à la fin des années 1970, Pierre Mayrand créa les premiers écomusées du Québec, inspirés des écomusées français, à partir d'une première expérience appuyée sur le patrimoine régional, en Haute-Beauce, en 1979. L'éveil de la nouvelle muséologie (comme l'a appelée René Rivard) dans cette région a été une " révolution tranquille » (Rivard, 1985, p. 202) - qui, progressivement, a relié la patrimonialisation à l'éducation populaire et au développement économique.

Une nouvelle muséologie a émergé dans le monde comme un processus de changement des valeurs qui a préparé le terrain pour la création d'un champ scientifique et politique. Généralement, un champ ne peut pas échapper aux luttes, plus ou moins inégales, entres des agents dominants et leurs concurrents, les « nouveaux entrants », considérés comme dominés (Bourdieu, 1976, p. 96). Cependant, dans le cas de la muséologie, on ne peut identifier un champ scientifique configuré au moment de l'apparition des idées dites "hétérogènes" sur l'objet central qui constitue un capital scientifique spécifique. Au lieu de transformer un champ existant, en fait, cette pseudo-révolution a inauguré le champ scientifique qu'elle a inventé comme ancien.

Plus qu'un champ de grandes révolutions, la muséologie s'est constituée par d'« innombrables petites révolutions permanentes » (Bourdieu, 1976, p. 96) dont les rôles des dominants et des dominés n'ont jamais été très bien définis. Envisageant sa légitimation, la muséologie a commencé à réagir à une demande collective pour sa réinvention. Mais l'éveil d'une muséologie pensée différemment était conditionné par l'invention d'une muséologie traditionnelle dépassée. La muséologie, pour être pensée comme une autre muséologie,

encourageant des échanges de savoirs et de perspectives. Dans les années 1970, son travail avec les écomusées allait témoigner de son avis alternatif par rapport à un système exclusif sur les musées en France. 
« n'est apparue nouvelle que dans la mesure où la muséologie avait vieilli » (Desvallées, 1992, pp. 22-23).

\section{De l'écomusée du Creusot à la Muséologie (nouvelle)}

Le $1^{\text {er }}$ septembre 1971 est née au Creusot la Maison de l'Homme et de l'Industrie, qui deviendra le prototype d'une nouvelle expérience muséale. II s'agissait fondamentalement de faire que le public (la communauté urbaine) s'approprie son propre patrimoine, qui était révélé dans le même moment que la création d'un musée. Comme un prototype développé par un groupe de professionnels français au Creusot, l'écomusée, créé plus tard à partir de ce modèle, est devenu un concept clé de la muséologie, théorisé et testé empiriquement au sein de cette discipline nouvelle, et, plus récemment, emprunté à d'autres sciences qui vont aussi réfléchir sur cette notion d'un modèle de musée réinventé.

Les notions de «participation de la collectivité » ou d'« identité culturelle » (Mairesse, 2002, p. 101) ou l'idée de " communauté » ou de "groupe social muséalisé » (Brulon Soares, Scheiner \& Campos, 2010) se sont instrumentalisées d'abord dans le contexte des pays industrialisés et ensuite dans les pays en développement. La chaîne de transformations qui a encadré les musées et la muséologie dans le monde à partir de la nouvelle muséologie et de l'écomuséologie, comme on l'a déjà démontré, a été un mouvement centralisé dans le contexte européen.

En tant que concept français créé par Hugues de Varine et théorisé par Georges-Henri Rivière dans les années 1970, l'écomusée est responsable de la prolongation et du renforcement de nouvelles expérimentations de l'activité muséale. Les premiers écomusées sont perçus comme des «laboratoires » (Rivière, 1985, pp. 182-183) pour amener les collectivités à se connaître mieux et à se reconnaître par l'appropriation d'un patrimoine, ainsi que par la renégociation d'un passé. L'écomusée traduit aujourd'hui un modèle ou contre-modèle - clé pour penser la muséologie ; il a été conçu comme un objet de rupture avec la muséologie dite traditionnelle, avant de lui être incorporé.

Pour les penseurs de la muséologie, l'Écomusée de la communauté urbaine du Creusot-Montceau-les-Mines créé entre 1973 et 1974 a été la première expérience visant à changer le sens du mot «musée ». Dans un texte publié en 1973, écrit par Hugues de Varine, sur l'activité muséale au Creusot, l'écomusée a servi « de prétexte et de support à la réflexion théorique sur des traits essentiels de la nature du musée moderne " (Varine-Bohan, 1973, p. 243). Au Creusot, quelques revues dédiées à l'étude des musées ont été créées ou ont changé leurs perspectives: c'est le cas de CRACAP / Informations, édité à partir de la création du Centre national de Recherche d'Animation et de Création pour les Arts Plastiques qui a précédé l'écomusée, et de la revue Milieux, éditée à partir de la fin des années 1970.

Au-delà de la participation de Varine et Rivière, invités à inventer un nouveau modèle qui est devenu l'écomusée, l'originalité de cette entreprise a attiré beaucoup de visiteurs (français et étrangers) désirant s'en inspirer (Bellaigue, 2012) ${ }^{33}$. Pendant la seconde moitié

\footnotetext{
${ }^{33}$ Communication verbale. Entretien à Paris, 5 avril 2012.
} 
des années 1970 et le début des années 1980, l'écomusée du Creusot a attiré l'attention d'un grand public de muséologues ou de professionnels des musées traditionnels, ce qui a suscité un changement effectif des mentalités. L'écomusée a donc fonctionné comme un énoncé performatif qui visait à faire advenir ce qu'il énonçait (Bourdieu, 1981, p. 69) - à savoir l'objet d'une science en tant qu'objet social - depuis le moment où le mot a été créé et même après quand les premières pratiques servirent à la conception de toute une théorie et à la configuration d'un champ politique ${ }^{34}$.

En contact permanent avec Rivière et en charge des galeries du Musée national des Arts et Traditions populaires supervisées par celui-ci entre 1970 et 1975, Desvallées est devenu un bon connaisseur des entreprises écomuséologiques, alors aux premiers stades du développement des écomusées en France. Selon lui, pendant sa vie professionnelle Rivière avait comme préoccupation principale celle de la création des écomusées dont il mûrissait le concept depuis les années $1930^{35}$ (Desvallées, 2014). C'est à partir de la naissance des parcs naturels, dans le cadre du ministère de l'Environnement, que cette idée prit corps.

Envisageant une muséologie pratique et expérimentale née au milieu de cette chaîne de transformations sur l'idée du "musée ", Desvallées (1981) a proposé, dans l'article " Muséologie (nouvelle) » écrit en 1980 pour le supplément de l'Encyclopaedia Universalis (repris dans l'édition de 1985 et les suivantes jusqu'à nos jours), le terme qui a inspiré l'association Muséologie nouvelle et expérimentation sociale (MNES), créée en $1982^{36}$, et la nouvelle muséologie en tant qu'un mouvement international chez l'ICOFOM et le MINOM à partir de 1983. Pour l'auteur de cette célèbre expression :

C'était la tâche que je me proposais donc : résumer tout le mouvement de rénovation qui avait pris naissance une douzaine d'années plus tôt. [...] Alors j'ai pensé profiter un peu par jeu parodique, par provocation - de la mode qui abusait alors des "nouvelles" doctrines: on avait la "nouvelle philosophie", la "nouvelle économie", la "nouvelle histoire", etc. Mais, comme je ne tenais pas spécialement à publier un manifeste, j'intervertis les termes et me contentai, derrière "muséologie", de placer "nouvelle" entre parenthèse » (Desvallées, 1985, p. 66).

\footnotetext{
${ }^{34}$ II faut souligner que, dans un champ de pouvoir plus vaste et dominant, comme décrit par Pierre Bourdieu, les muséologues et les professionnels des musées étaient une "fraction dominée de la classe dominante ", ce qui explique la quête pour la légitimation d'un champ scientifique spécifique. Cf. Bourdieu, Pierre \& Wacquant, Löic. Réponses. Pour une anthropologie réflexive. Libre Examen - Politique. Paris: Éds. du Seuil, 1992.

${ }^{35}$ Sa conception des années 1930 était plutôt celle des musées de plein air, dont il a intégré le modèle dans le modèle d'écomusée pensé par Hugues de Varine. C'est ce qui explique le malentendu et (parfois) le double langage qui règne lorsqu'on parle des écomusées.

${ }^{36}$ L'association MNES a été créée par un groupe de conservateurs provoqués par l'article de Desvallées dans l'Universalis, en 1982. Dans un premier moment, Evelyne Lehalle, qui était au musée d'Histoire de Marseille, ainsi qu'Alain Nicolas, qui dirigeait le musée, ont lu ses idées. Sur la MNES, une grande partie de ses idéalisateurs n'ont pas beaucoup publié sur la nouvelle muséologie. C'est le cas d'Evelyne Lehalle (la fondatrice et première présidente de l'association), de Marie-Odile de Barry (qui a travaillé au Creusot), de Françoise Wasserman (qui créa l'écomusée de Fresnes et fut la seconde présidente de la MNES), d'Alexandre Delarge, de Sylvie Douce de la Salle, de Joëlle Le Marec et d'autres (Desvallées, 2014). Certains, néanmoins, ont eu une grande pratique.
} 
Pour Desvallées, le sens de la nouveauté dans l'expérience muséale était déjà présent dans l'action et les écrits de Rivière et de Varine, en tant que directeurs de l'ICOM - le premier à partir de 1946, le second à partir de 1965 (Desvallées, 1992, p. 15). En effet, Varine avait déjà utilisé le terme "nouvelle muséologie » pour la première fois, mais pas avec la même accentuation que Desvallées, deux ans avant, pour désigner une opposition à la «muséologie traditionnelle », dans un texte de 1978 sur les fondements de l'idée d'écomusée ${ }^{37}$. Aujourd'hui, les anciens objectifs des écomusées sont partagés par beaucoup d'autres musées, comme les musées d'ethnologie et les musées d'art contemporain. Pour ces autres musées qui ont été "communautarisés ", le sens de la communauté a beaucoup varié au long des dernières années et dans les différentes expériences. Les musées sont sortis de leurs murs et de leur centralité sur les collections, et la muséologie a trouvé dans l'expérience muséale, en tant qu'expérience sociale, son vrai objet d'étude.

\section{L'organisation d'un Mouvement International}

À partir de 1971, à la demande de ses membres et à l'initiative de son nouveau président, Jan Jelínek, I'ICOM fut réorganisé. Le fait que ce fut au moment de la Conférence Générale, au vu des demandes des membres pour une organisation plus adaptée à toutes les nécessités des différents contextes muséaux et sociaux, est significatif. Les membres des États-Unis expriment le sentiment largement répandu que "I'ICOM ne répond pas aux besoins de l'ensemble de ses membres" (Nouvelles de l'ICOM, 1971, p. 17). C'est aussi le moment où les Africains proposent la création de I'Union des musées africains, appuyée par l'ICOM. L'Amérique Latine, "en vue de la renaissance des musées », demande à l'ICOM de considérer le musée " comme organisme social » afin qu'il puisse satisfaire "les besoins de la société qu'il sert » (Nouvelles de I'ICOM, 1971, p. 23). Ces sont des revendications qui ont été reprises, un an plus tard, à la réunion de Santiago du Chili, organisée par l'UNESCO.

Les années 1970 marquent aussi le moment des premiers signes d'une formation effective à la « muséologie », en tant que « discipline indépendante " (Nouvelles de I'ICOM, 1971, p. 20). Pendant la Conférence de 1971, un groupe, présidé par Rivière, organise une réunion à la demande de I'UNESCO, pour réfléchir à l'élaboration d'un Traité de Muséologie, « ayant pour but de définir les principes et les méthodes de la muséologie » (Nouvelles de l'ICOM, 1971, p. 20). En 1972, l'ICOM propose le développement d'un groupe de travail pour la préparation d'une terminologie muséologique au sein du Comité international pour la formation du personnel (ICTOP) (Nouvelles de I'ICOM, 1972, p. 20). Cette tâche deviendra finalement, à la fin de cette décennie, une tâche de l'ICOFOM, comité qui avait été créé en 1977 pour l'étude de la muséologie ${ }^{38}$.

Progressivement, la [pseudo-]révolution - qui était, au début, plutôt une révolution sociale au sein du musée - a été perçue comme une

\footnotetext{
37 La « nouvelle muséologie », selon Varine, serait une muséologie « d'origine et d'essence communautaire » (Varine, 1978, pp. 446-487).

38 Après plusieurs années de travail au sein de l'ICOFOM, organisée par André Desvallées et François Mairesse, cette tâche a eu son plus récent accomplissement avec la publication, en 2011, avec le soutien de l'ICOM, du Dictionnaire Encyclopédique de Muséologie.
} 
révolution scientifique entre les agents de la muséologie mondiale. Si le musée n'est pas seulement un établissement, et il peut aussi être un laboratoire, une expérience, un instrument pour les communautés, il peut se développer dans un mouvement continu, comme un phénomène social (Scheiner, 2007, p. 154). Et si la nouvelle muséologie a configuré un changement d'ordonnance dans la muséologie internationale - pas simplement par une rupture complète avec la muséologie précédente, mais à partir d'un engagement social et scientifique inédit -, on peut dire qu'elle a créé un champ de la muséologie ou muséologique par l'organisation de ses agents selon des idéologies spécifiques et les positions objectives qu'ils occupent. Par l'annonce d'une muséologie nouvelle, les dirigeants de ce mouvement complexe, d'abord en France, puis dans le reste du monde, ont fait exister ce qu'ils ont énoncé : la muséologie.

\section{La réinvention de la Nouvelle Muséologie}

Selon la définition qu'André Desvallées a donnée au mouvement, la nouvelle muséologie est restée "une école vivante de contestation », et, notamment en France, elle a été aussi, par nécessité, "un mouvement de résistance contre de véritables détournements de sens » (Desvallées, 1992, p. 16) de la muséologie et de la muséographie dites "officielles". Les années 1980 sont marquées par la rapide internationalisation du mouvement. Mais il est vrai, comme le considère l'auteur, que c'est sans doute dans les pays où le conservatisme muséal était le plus grand que le mouvement pour la nouvelle muséologie a trouvé le plus d'écho (Desvallées, 1985, p. 69). En général, les gens qui ont adhéré au projet de faire une muséologie différente, plus idéologique ou sociale, d'un côté, et plus théorique, de l'autre, étaient membres du MINOM et/ou de l'ICOFOM, où ils partageaient indifféremment les idées d'un groupe de penseurs et/ou d'un autre.

C'est à partir de ces deux forums de débat pour la muséologie internationale que l'organisation géopolitique d'un champ du savoir s'est configurée. Dans la réunion de l'ICOFOM, à Londres pendant la conférence générale de l'ICOM de 1983, les grands penseurs de la muséologie ${ }^{39}$ ont été confrontés à l'idée de la configuration d'un champ scientifique et politique. A ce moment-là, l'ICOFOM était à un "tournant", en quête d'une plus grande participation de ses membres (Sofka, 1983, p. 2). Les participants des conférences scientifiques d'ICOFOM à Londres ont été nombreux - entre 85 et 100 (Nouvelles muséologiques, 1984, p. 4) - et ses membres, en 1983 , composaient un ensemble de 115 professionnels, répartis entre 39 nationalités avec une grand majorité d'Européens, parmi lesquels des Français, des Allemands, de l'Ouest et de l'Est, des Russes, des Tchèques, des Danois, des Hollandais et quelques Anglais, des Américains du Nord, dont une majorité de Canadiens et quelques membres des Etats-Unis, des Américains de l'Amérique Latine, dont des Brésiliens, des Mexicains, des Argentins et des Asiatiques, dont quelques Japonais et des Indiens.

Cette même année, à Londres, sur proposition notamment du Canadien Pierre Mayrand, le Bureau exécutif a été chargé de prendre une décision en ce qui concernait la création d'un sous-

\footnotetext{
${ }^{39}$ Ont participé à cette conférence André Desvallées, Mathilde Bellaigue, Vinoš Sofka, Zbynek Stránský, Waldisa Rússio, Tereza Scheiner, Flora Kaplan, entre autres.
} 
groupe de travail sur la « muséologie communautaire » (Sofka, 1984, p. 12). Le débat pendant la conférence a été compliqué par des problèmes de langue, voire de mentalité, et les Français ont été soutenus par les Canadiens français, les Belges, les Espagnols et plus généralement ceux que l'on appelle les Latins (englobant les Sud-Américains et les Portugais). Les anglophones, ne comprenant pas ce que souhaitaient les francophones, refusèrent que soit créé, à l'intérieur du Comité, ce groupe de travail pour les écomusées et musées communautaires dont les représentants québécois proposaient la création. Du coup, les Québécois prirent l'initiative de provoquer, un an après, une première réunion internationale sur le même thème. Le groupe dirigé par Mayrand a organisé une réunion à Québec et à Montréal entre le 8 et le 13 octobre 1984, d'où est sortie la demande d'un nouveau comité (Desvallées, 1985, p. 67). En 1985, le MINOM a été officialisé dans une réunion à Lisbonne par ces mêmes Canadiens, et quelques autres Européens. Un champ de négociations politiques et d'échange d'idées était né.

\title{
Le MINOM et la socio-muséologie
}

À partir de l'Atelier organisé à Québec, en 1984, pour discuter les principes de base d'un mouvement de la nouvelle muséologie, s'est imposée l'idée «de créer une association ou une fédération internationale pour la nouvelle muséologie et de demander sa reconnaissance à I'ICOM et à l'ICOMOS comme association affiliée " (Déclaration de Québec, 1984). Après le rapport fait par André Desvallées, en 1985, au bureau de I'ICOM (Desvallées, 1985), ce dernier accepta le MINOM comme organisation associée. Dans cette même année, à Lisbonne, une Assemblé générale a officialisé le mouvement qui ouvrit son champ d'action en tant que mouvement organisé :

\begin{abstract}
Le MINOM reconnaît comme représentatifs de ce mouvement des musées, des réalisations et des actions individuelles ou collectives pouvant prendre des formes variées suivant les pays et les situations particulières; les écomusées, de même que les musées de voisinage, en sont les exemples les mieux connus. Le mouvement englobe de nombreuses autres réalisations et actions, plus ou moins structurées, mais qui présentent les mêmes caractères (MINOM, Règlements généraux, 1985).
\end{abstract}

Tenant sa première assemblée à Lisbonne, à l'occasion du 2ème Atelier International de la Nouvelle Muséologie, le groupe majoritaire des francophones qui avaient formé le MINOM a reçu, même au début, de nombreuses nouvelles demandes d'adhésion, surtout de membres portugais et de quelques Espagnols (MINOM, Demandes d'adhésion, 1985). Parmi les membres français, quelques membres fondateurs de la MNES participèrent à la création du MINOM - c'était le cas d'Evelyne Lehalle. Mais, peu à peu, avec une plus grande participation des Latino-Américains pendant les années 1990, ainsi que de Portugais, d'Espagnols et de quelques Africains, le mouvement, qui était formé, au début, de Canadiens du Québec et de quelques Européens (surtout de Français), a trouvé une nouvelle place dans la muséologie mondiale.

Ce qui a donné de l'importance à la voie muséologique du MINOM, c'est la variété des expériences considérées comme "nouvelles " (écomusées, musées de voisinage, musées locaux, etc.), outre un langage qui s'est intéressé directement aux problèmes sociaux au 
sein des musées. En effet, c'est pour cette dernière raison que les professionnels des pays en voie de développement se sont identifiés avec le mouvement, et c'est dans ces pays que les nouvelles tendances d'une muséologie plus "sociologisée " ont trouvé leurs racines. Les "nouveaux musées " qui ont été créés dans ces régions, surtout à partir des années 1990, ont renouvelé les muséologies locales et leurs répercussions sur les contextes socioculturels de ces pays. Dans ces contextes, la formule (appropriée) des musées communautaires était mieux adaptée que celle des musées traditionnels introduits par la colonisation.

À Québec, en 1984, les penseurs de la nouvelle muséologie ont insisté sur le rôle interdisciplinaire de la muséologie (Déclaration de Québec, 1984) par rapport à ses théories et méthodes. Plus tard le MINOM a proposé au champ muséologique international une "muséologie sociale", ce qui sous-entend la transition vers un musée plus ouvert aux sociétés humaines et aux problèmes sociaux. Le MINOM va donc démarquer un champ de débats autour de cette muséologie émergente. Selon Mário Moutinho, le penseur portugais qui est devenu la voix principale de la "muséologie sociale " ou " socio-muséologie », l'élargissement de la notion de patrimoine conduit à la redéfinition de "l'objet muséologique » et entraine la participation des groupes dans la gestion des pratiques muséales, l'utilisation de la muséologie dans le développement des sociétés, l'interdisciplinarité, l'utilisation des nouvelles technologies et la muséographie comme un moyen de communication autonome (Moutinho, 1993, p. 8).

Dans cette perspective sociale, l'emploi des néologismes comme "nouvelle muséologie ", "muséologie active ", "muséologie ouverte ", etc., utilisés pour couper avec un ordre "dépassé ", relève d'une tendance qui, empiriquement, ne représente pas vraiment une nouveauté structurelle du champ de la muséologie. Plus qu'une branche théorique ou une tendance révolutionnaire, le MINOM a développé un discours décentralisé sur la pratique muséale, mais le mouvement n'a pas démontré un contrôle sur ses appropriations dans les différents contextes socio-muséologiques où il était appliqué.

\section{La pensée muséologique de l'ICOFOM}

$\mathrm{Si}$, à l'intérieur du MINOM à la fin du $\mathrm{XX} \mathrm{X}^{\mathrm{e}}$ siècle, le caractère social et éthique du musée a été souligné dans les écrits produits sous la forme de manifestes idéologiques, pour l'ICOFOM la production théorique sur le même sujet s'est présentée sous une forme plus académique (Scheiner, 2007, p. 156). Aujourd'hui on peut identifier un corpus théorique produit dans le comité, lié à la nouvelle muséologie, à travers des travaux comme ceux d'André Desvallées, de Mathilde Bellaigue, d'Hugues de Varine, de Marc Maure, de Jean Davallon, parmi les auteurs francophones, de Waldisa Rússio Guarnieri, de Tereza Scheiner, de Norma Rusconi, de Nelly Decarolis, parmi les Latino-Américains, et un peu dans les travaux de quelques auteurs de l'ancienne Europe de l'Est, comme Tomislav Sŏla et Ivo Maroević.

Les documents produits par I'ICOFOM entre 1979 et 1989 définissent les bases de ce qu'aujourd'hui on reconnaît comme la théorie de la muséologie. Le thème du premier numéro de la publication intitulée Documents de Travail sur la Muséologie 
$(\text { DoTraM })^{40}$, en 1980, a posé la question « La muséologie - science ou seulement travail pratique du musée ? ", en proposant déjà la possible existence d'un champ proprement scientifique. Cette publication a marqué le passage de la production de textes sur les musées vers la production effective de textes sur la muséologie. Plus tard, I'ICOFOM Study Series (ISS), la principale publication du comité jusqu'à nos jours, a eu ses premiers numéros présentés à Londres, en 1983, avec un thème plus épistémologique, « La méthodologie de la muséologie ${ }^{41}$ », et l'autre, plus social, « Musée territoire - société ${ }^{42} »$. Dans les années 1980, l'ICOFOM a insisté sur l'importance de visions théoriques et de thèmes sociaux avec « Muséologie et identité » (1986) et « Muséologie et pays en voie de développement - aide ou manipulation ? » (1988). La constitution de la muséologie comme un champ de savoir reconnu, à partir de visions plus ouvertes de son objet, est devenue un des points axiaux du comité :

« Le Comité international de I'ICOM pour la muséologie (ICOFOM) a donc décidé de consacrer sa réunion annuelle de septembre 1987 à Espoo, Finlande, à l'étude du muséeinstitution dans une nouvelle perspective plus large, non seulement au nom du développement de la muséologie en tant que discipline scientifique, mais également en tant que contribution au développement du champ d'intérêt muséologique en général » (van Mensch, 1988, p. 5).

Au long des années 1990, de nombreux membres de l'ICOFOM ont publié des livres emblématiques sur la pensée de la nouvelle muséologie : c'est le cas de Vagues: une anthologie de la Nouvelle Muséologie (Desvallées, De Barry \& Wasserman, 1992), coordonné par Desvallées, ou de l'œuvre de Jean Davallon sur l'environnement naturel et les musées (Davallon, Grandmont \& Schielle, 1992). Les décennies 1990 et 2000 sont marquées par la diffusion des idées de la nouvelle muséologie et de la notion d'écomusée entre des chercheurs d'autres champs du savoir : c'est le cas des travaux du sociologue Henri-Pierre Jeudy (1990) ou des recherches des ethnologues comme Octave Debary (2002), Jean-Louis Tornatore (2003) et Serge Chaumier (2004). L'ICOFOM a dialogué avec ce champ de recherches, actif jusqu'à présent.

À partir de la recommandation de l'ICOM pour la décentralisation et la régionalisation de ses comités internationaux, et du soutien de Vinoš Sofka, alors président de l'ICOFOM, s'est créé, en 1990, I'ICOFOM Latinoamérica, sous-comité régional de l'ICOFOM pour l'Amérique Latine et les Caraïbes. Conceptualisé par Tereza Scheiner et Nelly Decarolis, I'ICOFOM LAM a servi à la diffusion des idées et savoirs muséologiques dans le sous-continent (Decarolis, 2006, p. 3).

L'ICOFOM, qui a réuni un grand nombre de membres des pays en voie de développement, a présenté à l'intérieur de ses publications des visions hétérodoxes sur l'idée du musée au service des sociétés,

\footnotetext{
${ }^{40}$ Les DoTraM - ou, en anglais, les Museological Working Papers (MuWoP) - ont été la première publication de l'ICOFOM sur des questions muséologiques, éditée par Vinoš Sofka, entre 1980 et 1981. Le second numéro, paru en 1981, a traité du thème "Muséologie et interdisciplinarité ». Cf. DoTraM - Documents de Travail sur la muséologie, n. 1, "La muséologie - science ou seulement travail pratique du musée ?"Stockholm, Suède, 1980. I DoTraM - Documents de Travail sur la muséologie, n. 2, «L'interdisciplinarité en muséologie ». Stockholm, Suède, 1981.

${ }^{41}$ Cf. ICOFOM Study Series - ISS 1. "Méthodologie de la muséologie et la formation professionnelle », Londres, 1983.

${ }^{42}$ Cf. ICOFOM Study Series - ISS 2. « Musée - territoire - société », Londres, 1983.
} 
ou des aspects plus philosophiques de la muséologie. Nombre de ses membres, adeptes de la nouvelle muséologie, ont compris le mouvement plutôt comme un retour aux sources, dont les principes n'ont rien de révolutionnaire : "ils sont simplement la muséologie " (Desvallées, 1992, pp. 22-23). Dans ce sens, le «retour aux sources " n'est pas une quête pour "l'essence " philosophique du musée - comme l'ont théorisé au début certains membres d'ICOFOM - mais plutôt une stratégie pour la légitimation de la base d'un champ scientifique, ce qui veut dire de "la muséologie » dans les différents contextes mondiaux où le terme est utilisé.

Aujourd'hui on peut observer que plus la nouvelle muséologie disparaît - parce qu'elle n'est plus ressentie comme nécessaire plus la muséologie se légitime avec un capital spécifique, dans la forme de concepts propres, d'une théorie et de méthodes plus au moins reconnues.

\section{Vers un champ scientifique de la muséologie}

La muséologie est encore muséologie (nouvelle) - entre parenthèses - parce que la nouveauté de la muséologie représente, en fait, le début d'un champ scientifique et non sa réinvention. C'est pour cette raison que l'épithète "nouvelle " a été utilisée pour évoquer, dans les différents moments du développement de la pensée muséologique, ses différentes tendances et ses idéologies. En tant que champ pratique et professionnel qui habitait les musées pendant une grande partie du $X X^{e}$ siècle, la muséologie est devenue progressivement un champ scientifique, habitant l'université et les forums académiques internationaux.

Ce qu'on a proposé ici - et qu'a proposé André Desvallées avant nous -, c'est l'idée d'une "nouvelle muséologie " en tant que forme contestataire de penser la muséologie à partir d'un encadrement réflexif des pratiques muséales. Le mouvement, daté des années 1970 et 1980, n'est pas seulement une partie de l'histoire de la discipline, il est toujours vivant dans les discours critiques - parfois idéologiques - qui réinventent constamment de nouvelles formes de penser la muséologie et les musées, même au $\mathrm{XXI}^{\mathrm{e}}$ siècle. En effet, cette capacité critique est-elle ce qui fait de la muséologie une discipline scientifique apte à se récréer elle-même en regardant l'évolution de son objet?

Depuis les années 1980, l'ICOFOM - comprenant à la fois des membres nouveaux et d'anciens membres - a absorbé progressivement cette capacité autoréfléchissante héritée des premiers penseurs de la nouvelle muséologie. Quand une pratique devient capable d'élaborer une théorie évaluative sur elle-même, à partir d'un éloignement nécessaire entre le sujet/évaluateur et son objet d'étude, ce qui en résulte est l'expression réflexive d'une voie théorique et pratique d'un champ scientifique en ascension. La muséologie (nouvelle), en constant état d'expérimentation, n'est pas une branche de la muséologie, mais la possibilité de sa "scientifisation ».

Entre l'ICOFOM et le MINOM, la nouvelle muséologie a survécu aux affrontements politiques et aux transformations de la réflexion théorique sur la muséologie dans le monde. L'affirmation de Desvallées selon laquelle il se place entre ceux « qui estiment qu'il n'y a pas d'ancienne et de nouvelle muséologie, mais simplement de 
la mauvaise et de la bonne muséologie" (Desvallées, 1985, p. 69), a constitué la déclaration de l'existence d'une muséologie dont font partie les différents agents, de l'ICOFOM au MINOM. La concurrence possible entre ces deux branches de la muséologie contemporaine n'a jamais suscité de vraies ruptures. En effet, nombre de ses agents ont collaboré de manière continue avec les deux organismes. On ne peut pas oublier que nombre des théoriciens de l'ICOFOM ont participés à la fondation du MINOM et collaboré avec le mouvement dans ses premières années et même après, comme, par exemple, Pierre Mayrand, René Rivard, Hugues de Varine (figure emblématique du mouvement) et André Desvallées (qui l'a légitimé lors de sa création).

L'absence d'une publication périodique du MINOM n'a pas permis une centralisation des idées qui ont été appropriées de différentes manières et dans les différents courants. Cependant, d'un côté et d'une certaine manière, le travail du MINOM a garanti l'existence d'un champ politique dans lequel les débats autour de la pratique muséale ont eu lieu. La réflexion politique sur la pratique montre ainsi le caractère social et évolutif de l'objet de la muséologie. Ce changement idéologique sur le musée, au long des dernières décennies, va renforcer l'importance de la production d'une base théorique fondamentale sur les idées de "musée » et de « muséologie » dans l'ICOFOM.

Le champ scientifique, espace d'une lutte de concurrence pour le monopole de l'autorité scientifique (Bourdieu, 1976, p. 89), présente, dans le cas de la muséologie, la confrontation de quelque chose de nouveau avec quelque chose d'inventé comme ancien pour engendrer la croyance dans la nouveauté, et, donc, l'autorité de la nouveauté qui commence à se porter comme la voie dominante c'est-à-dire qu'elle devienne un «capital spécifique de reconnaissance scientifique » (Bourdieu, 1976, p. 95). Cette invention d'un ordre nouveau a légitimé le processus pseudorévolutionnaire, structurant le champ de la muséologie. Avant l'invention de l'opposition entre une muséologie nouvelle et l'ancienne muséologie le champ scientifique, tel qu'on le connait aujourd'hui, n'était pas configuré. Et même si la révolution, dont cet article a traité, a été une fausse révolution, le champ qu'elle a créé n'a jamais été faux. Ses agents, ses institutions et les relations entre les positions qu'ils occupent sont réels et ils produisent de vrais effets sur la muséologie actuelle à partir de la croyance de ces agents concurrents dans la muséologie existante.

\section{Références}

Baghli, S. A., Boylan, P., \& Herreman, Y. (1998). Histoire de I'ICOM (19461996). Paris : Conseil International des Musées.

Bourdieu, P. (1976). "Le champ scientifique ». Actes de la recherche en sciences sociales, 2, 2-3, 88-104.

Bourdieu, P. (1981). "Décrire et prescrire [Note sur les conditions de possibilité et les limites de l'efficacité politique]. " Actes de la recherche en sciences sociales, 38, La représentation politique -2 , 69-73.

Bourdieu, P., \& Wacquant, L. (1992). Réponses. Pour une anthropologie réflexive. Libre Examen - Politique. Paris : Éd. du Seuil.

Brulon Soares, B. C. (2011). «O rapto das musas: apropriações do mundo clássico na invenção dos museus [L'enlèvement des Muses: appropriations du monde classique dans l'invention des musées]. ॥ Anais do Museu Histórico Nacional, Rio de Janeiro, 43, 41-65. 
Brulon Soares, B. C., Scheiner, T. C. M., \& Campos, M. D. (2010). « Sobre comunidades e museus: do gueto ao grupo social musealizado. [Sur les communautés et les musées : du ghetto au groupe social muséalisé.] » In XI Encontro Nacional de Pesquisa em Ciência da Informação, 2010, Rio de Janeiro. Anais do XI ENANCIB - Tema: Inovação e inclusão social: questões contemporâneas da informação.

Cameron, D. (1971). «The museum, a temple or the forum. » Curator, 14(1), 11-24.

Clair, J. (1976). «Les origines de la notion d'écomusée. » Cracap Informations, 2-3, 2-4.

Chaumier, S. (2004). "Écomusées: entre culture populaire et culture savante. " POUR, Dossier Mémoires partagées, mémoires vivantes, $181,65-70$.

Davallon, J., Grandmont, G., \& Schielle, B. (1992). L'environnement entre au Musée. Collection Muséologies. Lyon : Presses Universitaires de Lyon.

Debary, O. (2002). La fin du Creusot ou L'art d'accommoder les restes. Paris: CTHS.

Decarolis, N. (Dir.). (2006). El pensamiento museológico latinoamericano. Los documentos del ICOFOM LAM. [La pensée muséologique latino-américaine. Les documents de l'ICOFOM LAM.] Córdoba: ICOFOM LAM.

Déclaration de Québec (1984). Principes de base d'une nouvelle muséologie, Adoptée par le $1^{\text {er }}$ Atelier international Écomusée / Nouvelle muséologie, Québec, le 12 octobre, 1984. Consulté le 15 avril 2015 à partir de http://www.minom-icom.net

Desvallées, A. (1981). « Muséologie (nouvelle). » Encyclopaedia universalis, Supplément, t.2., 958-961 (6 colonnes).

Desvallées, A. (1985). "Muséologie nouvelle 1985. " Nouvelles muséologiques, septembre, 1985, Bulletin semestriel du comité international de l'ICOM pour la muséologie, Stockholm, 8, 65-69.

Desvallées, A. (1992). "Présentation. " Dans A. Desvallées, M. O. De Barry \& F. Wasserman (dir.). (1992). Vagues : une anthologie de la Nouvelle Muséologie (vol. 1). (pp.15-39). Savigny-le-Temple: Éditions W-M.N.E.S.

Desvallées, A., De Barry, M. O., \& Wasserman, F. (dir.). (1992). Vagues : une anthologie de la Nouvelle Muséologie (vol. 2). Savigny-leTemple : Éditions W-M.N.E.S.

Desvallées, A. (2014) In Brulon Soares, B. C. André Desvallées : entre muséologies (Entretien). ICOFOM Study Series - hors-série, pp.274-293.

DOTRAM - Documents de Travail sur la muséologie n. 1 (1980). « La muséologie - science ou seulement travail pratique du musée ? » Stockholm, Suède.

DOTRAM - Documents de Travail sur la muséologie n. 2 (1981). "L'interdisciplinarité en muséologie. " Stockholm, Suède.

ICOFOM Study Series - ISS 1 (1983). « Méthodologie de la muséologie et la formation professionnelle », Londres.

ICOFOM Study Series - ISS 2 (1983). "Musée - territoire - société », Londres.

Jeudy, H. P. (dir.). (1990). Patrimoines en folie. Paris : Éd. De la Maison des sciences de l'homme.

Kinard, J. R., \& Nighbert, E. (1972). The Anacostia Neighborhood Museum. Smithsonian Institution, Washington, D.C. Museum, The fine arts museum of Expo'70, Osaka, Paris, UNESCO, XXIV, 2, 103-108.

Principes de base pour le musée intégral. (1973). Museum International, Rôle du musée dans l'Amérique latine d'aujourd'hui, Paris, XXV, 3.

Mairesse, F. (2002). Le musée temple spectaculaire. Paris : Presses Universitaires de Lyon.

MINOM - Mouvement International pour une Nouvelle Muséologie. (1987). Le bulletin du MINOM, Montréal, 1, 0.

Moutinho, Mário. (1993). "Sobre o conceito de Museologia Social [Sur le concept de Muséologie Sociale]. " Cadernos de Museologia, 1.

Nouvelles de l'ICOM. (1971). «Conseil international des musées » - ICOM, 24, 4, Consulté à partir de : gallica.bnf.fr/ICOM. 
Nouvelles de I'ICOM. (1972). "Conseil international des musées » - ICOM, 25, 1, Consulté à partir de : gallica.bnf.fr/ICOM.

Nouvelles muséologiques. (1984). (Bulletin semestriel du comité international de l'ICOM pour la muséologie). Stockholm, 5.

Rivard, R. (1985). "Les écomusées au Québec. » Museum International, XXXVII, 148, 202-205

Rivière, G.-H., et al. (1971). Notes sur la $9^{\text {ème }}$ Conférence générale de I'ICOM, Paris, le 13 septembre 1971, document ronéoté, communiqué par André Desvallées, Archives personnelles, Paris, 2013.

Rivière, G.-H. (1985). "Définition évolutive de l'écomusée. » Museum, (Images de l'écomusée), Paris, UNESCO, XXXVII, 148, 182-183.

Scheiner, T. (2007). "Musée et muséologie : définitions en cours. » Dans Mairesse, F. et Desvallées, A. (dir.). (2007). Vers une redéfinition du musée ? (pp.147-165). Paris : L'Harmattan.

Sofka, V. (1983). "Londinium ante portas. Quelques réflexions avant l'ouverture de l'ICOM 83. " Nouvelles muséologiques. Bulletin semestriel du comité international de l'ICOM pour la muséologie. Stockholm, 4, 2-3.

Sofka, V. (1984). "Compte rendu de la Sixième réunion annuelle et la Troisième Assemblée Générale de l'ICOFOM. " Nouvelles muséologiques (Bulletin semestriel du comité international de l'ICOM pour la muséologie). Stockholm, 5.

Tornatore, J.-L., \& Paul, S. (2003). « Publics ou populations ? La démocratie culturelle en question, de l'utopie écomuséale aux «espaces intermédiaires». " Dans Donnat, O. \& Tolila, P. (dir.). (2003). Le(s) public(s) de la culture, Politiques publiques et équipements culturels. (pp.299-308). Paris: Presses de Sciences Po, vol. II (cédérom).

van Mensch, P. (1988). "Muséologie et musées. " Nouvelles de l'ICOM, 41, 3. Consulté à partir de gallica.bnf.fr/ ICOM (Conseil international des musées).

Varine-Bohan, H. de. (1973). «Un musée éclaté : le Musée de l'Homme et de l'Industrie. " Museum, XXV, 4, 242-249.

Varine, H. de. (1978). "L'écomusée " (1978). Dans A. Desvallées, M. O. De Barry \& F. Wasserman (coord.). (1992). Vagues : une anthologie de la Nouvelle Muséologie (vol. 1). (pp.446-487). Savigny-le-Temple : Éditions W-M.N.E.S.

\section{Résumé}

L'article propose l'analyse historique de la « nouvelle muséologie ", à partir de ses antécédents. L'auteur du terme «Muséologie (nouvelle) » (1980), André Desvallées, a défini le mouvement comme un "retour aux sources" de la muséologie. À partir des idées fondatrices de la " nouvelle muséologie » et du concept clé de l'« écomusée » - créé par Hugues de Varine et théorisé par Georges-Henri Rivière -, l'article présente la thèse selon laquelle la «nouvelle muséologie » a inauguré, d'un côté, une muséologie critique pensée dans les centres académiques, et d'autre part, l'idéologie du musée en tant qu'instrument social. Le Comité international pour la muséologie (ICOFOM) et le Mouvement international pour une nouvelle muséologie (MINOM), les deux forums observés dans ce travail, montrent les différents modes d'appropriation des mêmes idées originelles. Si l'ICOFOM a travaillé, influencé par des idées de la nouvelle muséologie, vers l'évolution de la discipline comme une totalité (de questions, de sujets, et, finalement, de concepts), le MINOM, au contraire, a diffusé l'idée d'une muséologie fragmentée, entre l'ancienne et la nouvelle, mais, plus récemment, entre un branche d'une «muséologie sociale» (ou «sociomuséologie») et l'autre muséologie, moins concernée par les problèmes sociaux.

Mots clé : Muséologie, Nouvelle Muséologie, Écomusée, ICOFOM, MINOM. 


\title{
Abstract
}

\author{
Invention and reinvention of "New Museology" (nouvelle \\ museologie)
}

The paper aims to present the historical analysis of "new museology" and its antecedents. The creator of the term "Muséologie (nouvelle) » (1980), André Desvallées, has defined the movement as a "return to the sources" of museology. From the basic ideas that founded "new museology" and the key concept of the "ecomuseum" - created by Hugues de Varine and conceptualized by Georges Henri Rivière - the paper presents the thesis according to which "new museology" has inaugurated, on the one hand, a critical museology produced in academic centers, and, on the other hand, the ideology of the museum as a social instrument. The International Committee for Museology (ICOFOM) and the International Movement for a New Museology (MINOM), the two forums here observed, show different models of appropriation of the same original ideas. While ICOFOM has been working under the influence of the new museology ideas towards the development of a discipline in its integral form (concerning questions, themes and, finally, concepts), MINOM, on the other hand, has spread the idea of a fragmented museology, divided between the old and the new, but more recently divided between a branch of socialmuseology and the other museology, less concerned with social problems.

Key words: Museology, New museology, Ecomuseum, ICOFOM, MINOM.

\section{Resumen}

\section{La invención y la reinvención de la Nueva Museología}

El documento propone un análisis histórico de la "nueva museología" y sus antecedentes. André Desvallées, el creador del término «muséologie (nouvelle)» (1980), ha definido el movimiento como un "retorno a las fuentes" de la museología. A partir de las ideas básicas, que dieron fundamento a la "nueva museología" y del concepto clave de "ecomuseo" - creado por Hugues de Varine y conceptualizado por Georges Henri Rivière - este artículo propone una tesis de acuerdo a la cual "nueva museología inauguró, por un lado, una museología crítica elaborada en los centros académicos, y, por otro, una ideología de museo como instrumento social. El Comité Internacional para la Museología (ICOFOM) y el movimiento internacional de una nueva museología (MINOM), los dos foros aquí analizados, muestran diferentes modelos de apropiación de las mismas ideas originales. Mientras que el ICOFOM ha venido trabajando bajo la influencia de las ideas de la nueva museología, tendiente al desarrollo de una disciplina integral, (en lo concerniente a interrogantes, temas y, por último, conceptos), el MINOM, por otro lado, ha difundido la idea de una museología fragmentada, tensionada entre lo viejo y lo nuevo, y más recientemente entre una rama de la "museología social" (o "socio museología") y otra museología, con menos énfasis en la problemática social.

Palabras clave: Museología, Nueva museología, ecomuseo, ICOFOM, MINOM 